\title{
Canon y educación musical
}

\author{
Análisis de los programas de formación \\ en relación con los programas de concierto \\ en la región metropolitana de la Argentina*
}

\section{Fabián Beltramino}

[Universidad Nacional de Lanús]

\begin{abstract}
Resumen Este artículo sintetiza los resultados de dos investigaciones acerca del canon musical: una dedicada al relevamiento de la programación de conciertos, la otra al de la oferta académica de la formación musical. Se establece, así, una correlación entre el canon que surge de la performance y aquél que surge de la instancia formativa de aquellos que ejercen la práctica. El trabajo efectuado permite concluir que por más "abierta" que se plantee la dimensión conceptual, en la práctica, los contenidos de una educación musical basada en lo modélico y en el paradigma de lo consagrado y lo ejemplar están más que vigentes.

En cuanto a la pregunta acerca de si el repertorio es consecuencia de la formación del músico o es un factor que orienta esa formación, se propone una tercera posición, la alternativa especular, que implica aceptar que ambas dimensiones se encuentran atrapadas en una relación circular respecto de la cual resulta muy difícil pensar alguna clase de desvío.
\end{abstract}

Palabras clave: Repertoire -

Education · Program

\begin{abstract}
Summary This article synthesizes the results of two investigations about the musical canon: one dedicated to the survey of concert programming, the other to the academic offer of musical training. Thus, a correlation is established between the canon that arises from performance and that that arises from the formative instance of those who practice the practice. The work carried out allows us to conclude that no matter how "open" the conceptual dimension may be, in practice, the contents of a musical education based on the exemplary and the paradigm of the consecrated and exemplary are more than current.

Regarding the question of whether the repertoire is a consequence of the musician's training or is a factor that guides that training, a third position is proposed, the specular alternative, which implies accepting that both dimensions are trapped in a circular relationship with respect to from which it is very difficult to think of any kind of deviation.
\end{abstract}

Keywords: Repertoire ·

Education · Program

* "Canon y formación musical académica", Proyecto de Investigación financiado por la Universidad Nacional de Lanús durante 2017-2018. Equipo dirigido por Fabián Beltramino e integrado por Sergio García Murillo, Juan Alfonso Samaja y Florencia Figueroa Dibarboure. 
Todo empieza por pensar la relación conflictiva entre el público y las manifestaciones más radicales del arte musical. Continúa en la necesidad de explicar "por qué se escucha lo que se escucha" y "por qué se toca lo que se toca». Y ante la respuesta "porque es lo que se sabe tocar y escuchar», la dimensión formativa, tanto de receptores como de productores de música, la educación musical, se vuelve objeto fundamental.

El análisis de la experiencia estética ha demostrado que desde las instancias educativas básicas de esos sujetos que conformarán el público del arte se trabaja tanto con lenguajes altamente codificados como con obras modélicas, y que las competencias que se adquieren están vinculadas a lo existente y lo consagrado. ${ }^{1}$

El análisis de las programaciones de conciertos, por su parte, ha mostrado que el repertorio del siglo XIX acumula todavía hoy alrededor del $40 \%$ del repertorio, concentrado en la obra de poco más de diez compositores ${ }^{2}$, núcleo duro de ese "canon" asociable a un lenguaje previsible, en el que las expectativas que la obra va generando en el oyente muy probablemente se cumplen durante su transcurso. ${ }^{3}$

A partir de ello, la pregunta: el repertorio jes consecuencia y efecto de la formación del músico o es un factor que orienta esa formación?

Tomando como base dicho interrogante, se llevó a cabo el análisis de la oferta académica (documentos ministeriales, planes de las carreras, programas de las materias) de todos los niveles de la formación musical en el ámbito metropolitano de la República Argentina (Ciudad de Buenos Aires, Banfield, Lanús y La Plata): inicial (escuelas de música para niños), superior (ciclos superiores de los conservatorios), especializada (ciclos de licenciatura universitarios) e incluso aquella vinculada específicamente con la inserción profesional (preparación para concursos orquestales).

1 Beltramino, Fabián et al.: «La experiencia del arte: ¿acción social o acto privado? Hacia una teoría estético-sociológica de la recepción». Informe Final, Departamento de Humanidades y Artes - UNLa, 2013 [inédito].

2 Beltramino, Fabián: «La música en números. Relevamiento de la programación de conciertos: Permanencias, dominios y resistencias", en Revista Argentina de Musicología, n 15-16, Asociación Argentina de Musicología, Buenos Aires, ISSN: 1666-1060, 2016, pp.339-354.

3 Beltramino, Fabián et al.: "Código musical dominante. Cuestiones de economía cultural (capital, interés, valor)", Informe Final, Departamento de Humanidades y Artes - UNLa, 2017 [inédito]. 
El objetivo fue establecer una correlación entre el canon musical que surge de la performance, el concierto, y aquél que surge de la instancia formativa de aquellos que tienen a su cargo la ejecución musical.

La investigación que se describe ha conjugado dos áreas de conocimiento muy transitadas cada una de ellas, aunque no habitualmente vinculadas entre sí: educación musical y estudios sobre el canon.

Los trabajos dedicados a la educación musical, llevados adelante desde cierto sector de la musicología y, en mayor medida, desde la pedagogía o la psicología, focalizan por lo general las problemáticas del aprendizaje musical en sí mismo, contemplando aspectos vinculados fundamentalmente con la percepción o la psicomotricidad. Pocas veces se considera, con relación a estas dimensiones, la incidencia que pueda tener la elección de un determinado repertorio en lugar de otro.

El trabajo de Albert Casals y otros, por ejemplo, titulado «La concreción de un enfoque didáctico para la educación musical en Primaria» ${ }^{4}$, considera y refiere el repertorio que el docente elige y enseńa como una herramienta «abierta», tanto en lo que hace a la práctica misma como a la audición, aunque no reconoce las implicancias que conlleva, o acaso las orientaciones que establece en lo perceptivo, elegir entre un compositor u otro, entre una época u otra, o bien entre músicas occidentales o músicas de otras culturas, es decir, no reconoce los códigos específicos que se ponen a funcionar con cada género, estilo o clase de música que se pone a sonar, vinculados cada uno de ellos a matrices perceptivas específicas y no del todo intercambiables ni mutuamente accesibles.

Lo mismo ocurre en conceptualizaciones de la educación musical como herramienta de formación integral, basadas en la idea de educación «por» la música, como las que describe el trabajo de Silvana Longueira-Matos, titulado «Los retos educativos en la sociedad del conocimiento. Aproximación a las aportaciones desde el ámbito de la educación musical $»^{5}$. Se mencionan, con relación a este tipo de propuestas,

4 Casals, Albert et. al.: "La concreción de un enfoque didáctico para la educación musical en Primaria». Comunicación presentada en la Sesión IV del CEIMUS, Barcelona, publicado por Énclave Creativa, 2014, pp.586-594

5 Longueira-Matos, Silvana: «Los retos educativos en la sociedad del conocimiento. Aproximación a las aportaciones desde el ámbito de la educación musical». En TESI 14(3), 2013, pp.211-240. 
las exigencias y la atención que en dicha dinámica demanda el trabajo en grupo como parte del proceso de socialización, la dimensión comunicativa de la experiencia educativa efectivizada a través de lo artísticomusical, las dimensiones expresiva y sensorial de lo subjetivo involucradas en el trabajo, sin poner en consideración o hacer referencia a la música misma, a los objetos propiamente musicales con los cuales se va a trabajar en concreto, estableciendo, con relación a esa dimensión, un principio de transparencia o de identidad absoluta que permite un intercambio no problemático de dichos objetos; intercambio que parece no tener que responder por consideraciones estéticas, históricas o sociológicas específicas.

A partir de esto, se decidió establecer una sub-área tópica de la investigación: el cruce entre las propuestas programáticas y los programas, con sus fundamentaciones y objetivos (gran parte de ellos establecidos en función de una apertura y de una ampliación de horizontes y referencias), y el dictado concreto, con sus aspectos modélicos, normativos y ejemplares, casi siempre redundantes y afirmativos en torno a lo culturalmente dominante y aceptado. Al respecto, dos integrantes del equipo han producido un escrito que da cuenta de esta problemática específica. ${ }^{6}$

Los estudios sobre el canon, por su parte, de larga tradición en el ámbito anglosajón, parten del pionero trabajo de Joseph Kerman a comienzos de los8o, "Algunas variaciones canónicas»" y encuentran en William Weber a uno de sus mejores representantes. Uno de los trabajos que más se destaca en esta línea, sobre todo por el largo del período histórico que aborda, es el de T. Dowd, K. Liddle, K. Lupo y A. Borden, titulado "Organizando el canon musical: los repertorios de las principales orquestas sinfónicas de los Estados Unidos, de I842 a 1969».8

Luis Merino Montero, por su parte, en el trabajo titulado «Canon musical y canon musicológico desde una perspectiva de la música chilena»,

6 García, Sergio y Samaja, Juan Alfonso: “Canon musical y formación musical académica. El diseño curricular de las Escuelas de Música del GCBA». Ponencia a presentar en la XXIII Conferencia de la AAM/XIX Jornadas Argentinas de Musicología, La Plata, Agosto 2018 [inédita]

7 Kerman, Joseph: "A few canonic variations», en Critical Inquiry, v. 10 n`1, Septiembre, 1983

8 Dowd, T., Liddle, K., Lupo, K. Y Borden, A.: «Organizing the musical canon: the repertories of major U.S. symphony orchestras, 1842 to 1969". En Poetics, v.30, n¹-2, Mayo, 2002, pp.35-61 
ha abordado la construcción y el reconocimiento del canon musical, concibiendo a las instituciones musicales como agentes de comunicación. ${ }^{9}$

Mucho más cerca en el tiempo, aparece el trabajo de Pierre-Antoine Kremp, «Innovación y Selección: Orquestas Sinfónicas y la construcción del Canon Musical en los Estados Unidos (I879-1959)". ${ }^{10}$

En la conjunción de ambas dimensiones, donde los estudios previos son más bien escasos, uno de los trabajos de referencia es el de María Paz López-Peláez Casellas titulado «Una breve aproximación al canon musical en educación desde una perspectiva de género» ${ }^{11}$, abordaje que encuadra con la perspectiva iniciada por los trabajos «Género, profesionalismo y canon musical», de Marcia Citron ${ }^{12}$, y «Canon musical bajo fuego: el desafío de la musicología feminista», de Maree Mac Millan. ${ }^{13}$

El otro trabajo de referencia en el cruce entre canon y educación es el de Kindall-Smith y otros, titulado "Desafiando los paradigmas de exclusión en el canon musical tradicional: Implicaciones para la práctica de la educación musical $»^{14}$, en el que desde una pedagogía crítica, que apuesta por una responsabilidad social de la educación musical, se cuestiona la presencia y la incidencia del canon tradicional en el aula.

Lo que estos trabajos previos no problematizan, sin embargo, es la formación del músico, que se educa con relación a un horizonte profesional en el que el peso de lo convencional se impone con fuerza pero, al mismo tiempo, con total naturalidad.

Para describir esa formación, y luego poder compararla con el contenido de las programaciones musicales que hacen al ejercicio de la profesión musical, se llevó a cabo un desglose de las obras propuestas para

9 Merino Montero, Luis: "Canon musical y canon musicológico desde una perspectiva de la música chilena". En Revista Musical Chilena, v.60 n²05, Santiago, Junio, 2006, pp.26-33

10 Kremp, Pierre-Antoine: «Innovation an Selection: Symphony Orchestras and the construction of the Musical Canon in the United States (1879-1959)». En Social Forces, v.88 n³, 2010, pp.1051-1082

11 López-Peláez Casellas, María Paz: «Una breve aproximación al canon musical en educación desde una perspectiva de género". En Sophia, Universidad la Gran Colombia, v.9, 2013, pp.55-71

12 Citron, Marcia J.: "Gender, Professionalism and the Musical Canon». En The Journal of Musicology, Vol. 8, No. 1, winter, 1990, pp. 102-111

13 MacMillan, Maree: "Musical Canon under Fire: The Challenge of Feminist Musicology". En Australian Journal of Music Education, n¹, 1992, pp.5-12

14 Kindall-Smith, M. et al.: "Challenging exclusionary paradigms in the traditional musical canon: Implications for the music education practicen. En International Journal of Music Education, 29 (4), 2011, pp.374-386 
su estudio en los programas de enseñanza de las Escuelas de Música, la ESEAM "Juan Pedro Esnaola» y el Conservatorio Manuel de Falla de la C.A.B.A.; el Conservatorio «Gilardo Gilardi» de La Plata, el Conservatorio «Julián Aguirre» de Banfield, la Universidad Nacional de las Artes, la Universidad Nacional de La Plata y la Universidad Nacional de Lanús. ${ }^{15}$

Las materias consideradas, según instituciones y niveles específicos, fueron: Arpa, Bandoneón, Clarinete, Contrabajo, Corno, Dirección orquestal, Estudio orquestal, Fagot, Flauta dulce, Flauta traversa, Guitarra, Oboe, Percusión, Piano, Saxofón, Trombón, Trompeta, Tuba, Viola, Violín y Violonchelo. ${ }^{16}$

En cuanto a la sistematización de los datos, se organizó su digitalización en variables que permitieran ser puestas en relación, convirtiéndose así en indicadores susceptibles de ser leídos en sentidos diversos. Dichas variables fueron: Institución, Carrera, Materia, Año/Nivel, Compositor, Año de nacimiento del compositor, Obra, Año de estreno/ composición de la obra.

Se relevaron, en total, los programas de 97 materias, desglosados muchos de ellos en varios niveles, de los cuales surgió la indicación del estudio de 2236 obras. $^{17}$

A nivel general y, en promedio, lo que surge con claridad es la paridad entre los repertorios que se estudian y los que se ejecutan.

No hay diferencias significativas ni en lo que hace al año de nacimiento promedio de los compositores (I85I-I848, respectivamente) ni en cuanto al año promedio de composición de las obras (I86I-I867, idem).

La mayor concentración de los repertorios programados se da, en ambos casos, en el siglo XIX, apareciendo este dominio levemente más pronunciado en la programación de conciertos:

15 Para el estudio de las programaciones de conciertos fueron relevados los siguientes espacios, instituciones y cuerpos musicales a lo largo de cuatro temporadas completas (2010-2013): Teatro Colón (Orquesta Estable / Orquesta Filarmónica de Buenos Aires / Abono Bicentenario / Intérpretes Argentinos / Domingos de Cámara), Teatro Argentino de La Plata (Orquesta Estable), Facultad de Derecho, Auditorio de la Biblioteca Nacional, Mozarteum, Festivales Musicales, Academia Bach, Orquesta Sinfónica Nacional y Orquesta de la UNLa.

16 Se relevaron, en cada institución, los programas con desglose de obras de ejecución obligatoria.

17 Para el estudio de los programas de conciertos se relevaron 781 eventos, en los cuales se ejecutaron 2875 obras. 


\begin{tabular}{|c|c|c|c|}
\hline \multicolumn{4}{|c|}{ Concentración del repertorio por siglos } \\
\hline \multicolumn{2}{|c|}{ Educación } & \multicolumn{2}{|c|}{ Conciertos } \\
\hline s.XIII & 0 & s.XIII & 0,04 \\
\hline s.XIV & 0 & s.XIV & 0 \\
\hline s.XV & 0 & s.XV & 0,1 \\
\hline s.XVI & 0,23 & s.XVI & 1,06 \\
\hline s.XVII & 0,85 & s.XVII & 1 \\
\hline s.XVIII & 22,68 & s.XVIII & 17,38 \\
\hline s.XIX & 38,43 & s.XIX & 40,92 \\
\hline s.XX & 37,18 & s.XX & 34,29 \\
\hline s.XXI & 0,63 & s.XXI & 5,21 \\
\hline Total & $100 \%$ & Total & $100 \%$ \\
\hline
\end{tabular}

Figura I.

Concentración de repertorio por siglos

Como se ve, el repertorio de los siglos XVIII, XIX y xx, acumula, en ambos casos, más del 90\% del total (más del 99\% en educación), a partir de lo cual cualquier pretensión vinculada con el abordaje de «las músicas de todos los tiempos» queda de por sí descalificada en ambos ámbitos. Eso que desde la Ilustración ha pretendido establecerse con valor de universalidad queda, una vez más, puesto en evidencia como un constructo perfectamente historizable desde, justamente, el momento mismo del surgimiento de ese movimiento intelectual y político.

El factor concentración también opera, en ambos casos, con relación a los compositores programados.

En lo que hace a los conciertos, ya habíamos mostrado que los io compositores más programados (nacidos en promedio en I8I8) concentraban el $29 \%$ del total de obras.

En cuanto a los programas de educación, los io compositores más estudiados (nacidos en promedio en I799), concentran un porcentaje levemente menor, el $26 \%$ del total de obras:

\begin{tabular}{|c|c|c|c|}
\hline \multicolumn{4}{|c|}{ Compositores más programados y estudiados } \\
\hline \multicolumn{2}{|l|}{ Educación } & \multicolumn{2}{|l|}{ Conciertos } \\
\hline Mozart, Wolfgang A. & 1756 & Mozart, Wolfgang A. & 1756 \\
\hline Beethoven, L. van & 1770 & Beethoven, L. van & 1770 \\
\hline Bach, J. S. & 1685 & Brahms, J. & 1833 \\
\hline Brahms, J. & 1833 & Tchaicovsky, P. & 1840 \\
\hline Hindemith, Paul & 1895 & Bach, J. S. & 1685 \\
\hline Haydn, Franz Joseph & 1732 & Piazzolla, A. & 1921 \\
\hline Stravinsky, Igor & 1882 & Dvorak, A. & 1841 \\
\hline Tchaikovsky, P. I. & 1840 & Ginastera, A. & 1916 \\
\hline Weber, C. M. von & 1786 & Schumann, R. & 1810 \\
\hline Mendelssohn, F. & 1809 & Chopin, F. & 1810 \\
\hline
\end{tabular}

\section{Figura 2.}

Compositores

más programados

y estudiados 
En lo que hace al siglo XIX en particular, epicentro del repertorio canónico en ambos campos de estudio, es notable el nivel de concentración que presenta, en particular, a nivel compositores seleccionados.

Respecto del repertorio de conciertos, más del $50 \%$ de las obras programadas corresponde solo a Io compositores, todos europeos, la mitad alemanes (estrictamente 4 alemanes y i un austríaco: Schubert).

En el repertorio de estudio, por su parte, si bien este dominio se manifiesta en un porcentaje levemente menor ( $42 \%$ para los Io compositores más programados), resulta igualmente significativala recurrencia. Lo que aumenta para los programas educativos es la concentración geográfica, dado que comprende a 7 alemanes (6 alemanes más Schubert, nuevamente).

\begin{tabular}{|l|l|l|}
\hline \multicolumn{2}{l}{ Compositores del s. XIX más ejecutados y estudiados } \\
\hline Orden & Ejecutados en concierto & Estudiados \\
\hline 1 & Beethoven (alemán, 1770-1827) & Beethoven (alemán, 1770-1827) \\
2 & Brahms (alemán, 1833-1897) & Brahms (alemán, 1833-1897) \\
3 & Tchaikovsky (ruso, 1840-1893) & Tchaikovsky (ruso, 1840-1893) \\
4 & Dvorak (checo, 1841-1904) & Weber (alemán, 1786-1826) \\
5 & Chopin (polaco, 1810-1849) & Mendelsohn (alemán, 1809-1847) \\
6 & Schumann (alemán, 1810-1856) & Schumann (alemán, 1810-1856) \\
7 & Schubert (austríaco, 1797-1828) & Verdi (italiano, 1813-1901) \\
8 & Verdi (italiano, 1813-1901) & Dvorak (checo, 1841-1904) \\
9 & Bizet (francés, 1838-1875) & Wagner (alemán, 1813-1883) \\
10 & Mendelsohn (alemán, 1809-1847) & Schubert (austríaco, 1797-1828) \\
\hline
\end{tabular}

Figura 3. Compositores del siglo XIX más ejecutados y estudiados

Es evidente el modo en el que el ámbito musical local, tanto en lo que hace a la enseñanza como a la performance, reproduce casi a la perfección el canon europeo. Y el «casi» tiene que ver con la posición relevante que en la programación de conciertos adquieren Piazzolla y Ginastera. Por lo demás, se observa el mismo dominio por parte de los compositores clásico-románticos originarios del ámbito germano.

Con relación a la incidencia del repertorio del siglo xx en el canon, una incidencia no menor tanto en educación como en conciertos $(37 \%$ y $34 \%$, respectivamente), que permitiría pensar en una tendencia renovadora, es interesante señalar algo que afirman García y Samaja en el 
trabajo en el que comparan el diseño curricular con el repertorio propiamente dicho: este repertorio se incluye en tanto no entra en contradicción con el parámetro sonoro específico que el repertorio canónico ha naturalizado. ${ }^{18}$

Lo cual remite a algo ya afirmado también en un trabajo anterior: el canon no es tanto un conjunto cerrado de compositores y obras como un marco, un límite, con unas características bien definidas, que funciona como umbral de lo aceptable, de lo legitimable. ${ }^{19}$

$\mathrm{Si}$ algo distingue al repertorio que se estudia respecto del que se ejecuta, es la diversidad de compositores que presenta el repertorio del siglo $\mathrm{xx}$ que se incluye en los programas. Los compositores de este período representan el 57\% respecto del total de los compositores estudiados, mientras que los que se programan en concierto son apenas un $29 \%$ respecto del total, es decir, un espectro mucho más acotado, que se cierra aún más, dado que son apenas 26 compositores losque cubren prácticamente el 50\% del repertorio ejecutado con obras del período.

En lo que hace a las figuras dominantes, volvemos a encontrar en concierto la presencia de los argentinos Piazzolla y Ginastera, algo que en los programas educativos no sucede, siendo los cinco más estudiados de origen europeo.

Compositores del siglo XX
\begin{tabular}{|l|l|l|l|}
\hline Más ejecutados en concierto & \multicolumn{3}{l|}{ Más estudiados } \\
\hline Piazzola Astor & $1921-1992$ & Stravinsky lgor & $1882-1971$ \\
Ginastera Alberto & $1916-1983$ & Hindemith, Paul & $1895-1963$ \\
Ravel Maurice & $1875-1937$ & Ravel Maurice & $1875-1937$ \\
Debussy Claude & $1862-1918$ & Debussy Claude & $1862-1918$ \\
ShostakovichDmitri & $1906-1975$ & Bartók Bela & $1881-1945$ \\
\hline
\end{tabular}

Figura 4. Compositores del siglo xx 
Puede afirmarse, a partir de los resultados del relevamiento, que por más «abierta» que se plantee la dimensión conceptual, en la práctica, los contenidos de una educación musical basada en lo modélico y en el paradigma de lo consagrado y lo ejemplar están más que vigentes.

A partir de ello, la pregunta acerca de si el repertorio es consecuencia y efecto de la formación del músico o es un factor que orienta esa formación, podría responderse agregando una tercera posición, la alternativa especular, afirmando que ambas dimensiones se encuentran atrapadas en una relación circular respecto de la cual resulta muy difícil pensar en alguna clase de desvío.

Se estudia y se toca, en mayor medida, la música de compositores europeos nacidos, en promedio, a mediados del siglo xIx.

La concentración en ciertos compositores dominantes es clara tanto en los programas de enseñanza como en los de conciertos.

Es notable, en ambos relevamientos, el marcado dominio de compositores de origen o nacionalidad alemana.

Y mucho más notable es la ausencia de música compuesta en lo que va del siglo XXI que, si bien tiene una presencia mayor en las programaciones de conciertos $(5,21 \%)$ que en los programas de educación (o,63\%), ocupa un lugar muy menor respecto de la música de períodos anteriores en ambas dimensiones.

Esto vendría a corroborar no solo que la institucionalización siempre ocurre a posteriori, como es lógico, sino que (casi) siempre llega tarde, tanto en lo que hace a la potencia significante de las obras como con relación a la propia existencia de los compositores.

Si hubo un tiempo en el que la música que se escuchaba y la que se aprendía era la música de los contemporáneos, salvo la de algunos maestros del pasado a los que se dedicaba una atención especial en la etapa formativa, un tiempo en que se tocaba para y se aprendía de los que formaban parte de la comunidad musical a la que se pertenecía, la aparición del concierto público, como sabemos desde Henry Raynor ${ }^{20}$, cambió todo.

20 Raynor, Henry. (1986) 
Se separaron, por un lado, los roles de compositor e intérprete, y la edición musical permitió que las obras iniciaran un camino propio, en relación exclusiva con esa instancia que también el concierto hizo surgir, ese colectivo anónimo e incierto denominado público.

Ya no se compuso para alguien en especial, y ni siquiera se supo si lo que se componía iba a ser alguna vez ejecutado, ni ante quiénes.

El concierto propició la conformación de un repertorio que fue dando forma a eso que, a partir de un determinado momento, pasó a llamarse "música clásica» y que consiste, fundamentalmente, en poner a sonar la música compuesta por compositores muertos, que es lo que eufemísticamente vendría a pretender significar la expresión «del pasado».

Lógica necrofílica, sin más, que vuelve cada vez más difícil la posibilidad de entrar en contacto con la música del presente, la de los compositores vivos con los cuales se comparten las encrucijadas de un mismo tiempo histórico.

Lógica necrofílica de la que no escapa ni siquiera ese repertorio englobado en la etiqueta de lo contemporáneo, que ha dejado de ser una categoría cronológica para volverse, como lo moderno, una categoría estética que incluye su propio clasicismo, y en cuyos conciertos uno puede encontrar, perfectamente, músicas de casi un siglo de antigüedad compuestas por autores fallecidos hace décadas.

«Escucho gente muerta» o, más precisamente, «escucho alemanes muertos», podría concluirse parafraseando la frase del personaje de aquel famoso film ${ }^{21}$, más que adecuada para dar cuenta de la problemática que intentó describirse.

21 "Veo gente muerta», le decía el personaje del niño a su psicólogo en Sexto sentido, el film de M. Night Shyamalan, del año 1999. 


\section{REFERENCIAS BIBLIOGRÁFICAS}

BeLtRAMINO, fabián (2016): “La música en números. Relevamiento de la programación de conciertos: Permanencias, dominios y resistencias", en Revista Argentina de Musicología, no 15-16, Asociación Argentina de Musicología, Buenos Aires, ISSN: 1666-1060, pp.339-354.

BELTRAMINo, fabián et al. (2013): "La experiencia del arte: ¿acción social o acto privado? Hacia una teoría estético-sociológica de la recepción». Informe Final, Departamento de Humanidades y Artes - UNLa, [inédito].

Beltramino, fabián et al. (2017): “Código musical dominante. Cuestiones de economía cultural (capital, interés, valor)». Informe Final, Departamento de Humanidades y Artes - UNLa, [inédito].

CASALS, ALBERT et. al. (2014): "La concreción de un enfoque didáctico para la educación musical en Primaria". Comunicación presentada en la Sesión IV del CEIMUS, Barcelona, publicado por Énclave Creativa, pp.586-594.

Citron, Marcia J. (1990): «Gender, Professionalism and the Musical Canon», en The Journal of Musicology, Vol. 8, No. 1, pp. 102-111.

DOWD, T., LIDDLE, K., LUPO, K. Y BORDEN, A. (2002): «Organizing the musical canon: the repertories of major U.S. symphony orchestras, 1842 to 1969 ", en Poetics, v.30, no1-2, Mayo, pp.35-61.

GARCÍA, SERGIO Y SAMAJA, JUAN ALFONSO (2018): “Canon musical y formación musical académica. El diseño curricular de las Escuelas de Música del GCBA». Ponencia aceptada para la XXIII Conferencia de la AAM/XIX Jornadas Argentinas de Musicología, La Plata, [inédita].

KERMAN, JOSEPH (1983): "A few canonic variations", en Critical Inquiry, v.10n¹. KINDALL-SMITH, M. et al. (2011): "Challenging exclusionary paradigms in the traditional musical canon: Implications for the music education practice", en International Journal of Music Education, 29 (4), pp.374-386.

KREMP, PIERRE-ANTOINE (2010): «Innovation an Selection: Symphony Orchestras and the construction of the Musical Canon in the United States (1879-1959)", en Social Forces, v.88n³, pp.1051-1082.

LONGUEIRA-MATOS, SILVANA (2013): "Los retos educativos en la sociedad del conocimiento. Aproximación a las aportaciones desde el ámbito de la educación musical", en TESI 14(3), pp.211-240.

LÓPEZ-PELÁEZ CASELLAS, MARÍA PAZ (2013): “Una breve aproximación al canon musical en educación desde una perspectiva de género", en Sophia, Universidad la Gran Colombia, v.9, pp.55-71.

MaC millan, Maree (1992): "Musical Canon under Fire: The Challenge of Feminist Musicology", en Australian Journal of Music Education, n¹, pp.5-12. 
MERINO MONTERO, LUIS (2006): "Canon musical y canon musicológico desde una perspectiva de la música chilena", en Revista Musical Chilena, v.60n²05, Santiago, Junio, pp.26-33.

RAYNOR, HENRY (1986): Una historia social de la música: desde la Edad Media hasta Beethoven, Madrid: Siglo XXI.

WEBER, William (1989): "The Eighteenth-Century Origins of the Musical Canon", en Journal of the Royal Musical Association, v.114n¹, pp.6-17.

- (1994): "The Intellectual Origins of Musical Canon in Eighteenth-Century England", en Journal of the American Musicological Society, v.47n³.

(1999): «The History of Musical Canon», en Rethinking Music, Oxford: University Press, Cap.15, pp.336-355.

(2003): "Consequences of canon: The Institutionalization of Enmity between Contemporary and Classical Music", en Common Knowledge, Winter, 9 (1), pp.78-99. 